\title{
Hydrophilization of Amorphous Perfluoropolymer using Low-pressure Argon Plasma
}

\author{
Takao Ono $^{1,2}$, Takanori Akagi ${ }^{1,2,3}$, and Takanori Ichiki ${ }^{1,2,3}$ \\ 'Department of Bioengineering, School of Engineering, \\ The University of Tokyo, 7-3-1 Hongo, Bunkyo-ku, 113-8656, Japan \\ ${ }^{2}$ Core Research of Evolutional Science and Technology, Japan Science and \\ Technology Agency, 5 Sanban-cho, Chiyoda-ku, Tokyo, 102-0075, Japan \\ ${ }^{3}$ Center for NanoBio Integration, The University of Tokyo, \\ 7-3-1 Hongo, Bunkyo-ku, 113-8656, Japan
}

\begin{abstract}
The amorphous perfluoropolymer Cytop ${ }^{\mathrm{TM}}$ (Asahi Glass Co., Ltd.) is an attractive material for use in microdevices for biological and chemical analysis because of its high optical transparency, excellent chemical stability, and low refractive index of 1.34, which is almost the same as that of water. In this study, to increase the applicability of this polymer for such microdevices, the modification of its surface properties was investigated using low-pressure argon plasma. Hydrophilicity control with a water contact angle in the range from $30^{\circ}$ to $110^{\circ}$ and, moreover, the long-term stability of the hydrophilized surface were achieved for the first time. Although oxygen plasma treatment caused etching instead of hydrophilization, exposure to argon plasma led to bond breaking, the formation of free radicals, and finally the incorporation of hydrophilic groups as a result of the reaction with the oxygen or water from the atmosphere after the polymer was removed from the plasma reactor. Furthermore, to evaluate the suitability of this material for applications involving cell cultivation, the cell adhesion and proliferation properties of the plasma-treated surface were also investigated.
\end{abstract}

Keywords: amorphous perfluoropolymer, hydrophilization, argon plasma, cell adhesion

\section{Introduction}

In the field of micro total analysis systems $(\mu \mathrm{TAS})$ and biodevices, various transparent materials such as plastics, polymers, and glass have been used as substrate materials because of the importance of optical sensing and/or imaging in their application $[1,2]$. From this viewpoint, the amorphous perfluoropolymer Cytop ${ }^{\mathrm{TM}}$ (Asahi Glass Co., Ltd., Japan) is an attractive material, which is synthesized via a radial polymerization reaction from perfluoro-4-vinyloxy-1-butene. Its chemical structural formula is shown in Scheme 1. It has high optical transparency over a wide wavelength range from near ultraviolet (NUV) to near infrared (NIR) and excellent chemical stability. Moreover, it does not cause optical distortion due to the refractive-index mismatch at the interface with an aqueous solution because it has a refractive index of 1.34, almost the same as that of water [3]. When precise microscopy imaging is required for the analysis of biological cells or tissues, such optical distortion becomes an insurmountable obstacle [4].

Although Cytop ${ }^{\mathrm{TM}}$ is now applied commercially to optical fibers and as an antireflection layer of display panels [5], some researchers have

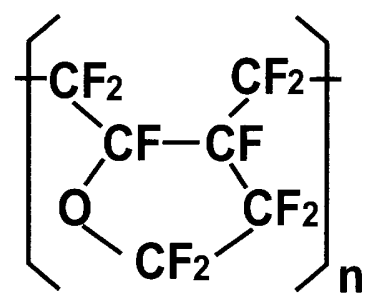

Scheme 1 Structural formula of amorphous perfluoropolymer Cytop ${ }^{\mathrm{TM}}$. 
also reported its use in $\mu$ TAS and biodevice research. They often utilize lack of reactivity with absorbed proteins or cells and its nontoxicity to living organisms in biological applications [6-8]. Recently, we have reported the anisotropic dry etching of this amorphous perfluoropolymer using low-pressure argon/oxygen mixture inductively coupled plasma with the aim of providing the process technology necessary for precise device fabrication using this polymer material [9].

On the other hand, Cytop ${ }^{\mathrm{TM}}$ is known to have a strongly hydrophobic nature comparable to that of poly(tetrafluoroethylene) (PTFE) with a large water contact angle of about $110^{\circ}$. Such a hydrophobic nature is sometimes undesirable for a biodevice material because most biodevices are required to handle wet samples in the microscopic space. Therefore, the development of a simple and robust surface modification technology for controlling the hydrophilicity of Cytop ${ }^{\mathrm{TM}}$ is required to increase its potential for use as a biodevice material.

With these as a background, in this study, we investigated the hydrophilicity control of the amorphous perfluoropolymer surface using low-pressure argon plasma. The changes in the surface properties of the polymer compared with those of untreated samples were examined by X-ray photoelectron spectroscopy (XPS), atomic force microscopy (AFM), and UV-visible (Vis)-NIR absorption spectroscopy to obtain a better understanding of the surface hydrophilization mechanisms. Furthermore, a cell cultivation experiment on the hydrophilized polymer surface was conducted to determine its cell adhesion characteristics and confirm its biological nontoxicity.

\section{Experimental Methods}

\section{1. Sample preparation}

A 3- $\mu$ m-thick Cytop ${ }^{\text {TM }}$ (CTL-809M) film coated on a borosilicate glass substrate of $20 \times 20$ or $30 \times 30 \mathrm{~mm}^{2}$ area and $0.5 \mathrm{~mm}$ thickness was used as a sample. After spin coating, the polymer film was cured at $50^{\circ} \mathrm{C}$ for $1 \mathrm{~h}$, after which the curing temperature was gradually raised for $1 \mathrm{~h}$ and finally maintained at $180^{\circ} \mathrm{C}$ for another $1 \mathrm{~h}$.

A bell-jar-type plasma reactor with a single-turn antenna wound around a 100-mmdiameter quartz bell jar and a stainless-steel chamber with a temperature-controlled inner wall were employed. Pure argon or $\mathrm{O}_{2}$ plasma was generated at a pressure of $4 \mathrm{~Pa}$. The $13.56 \mathrm{MHz}$ plasma source power was maintained at $25 \mathrm{~W}$ for argon plasma and $100 \mathrm{~W}$ for oxygen plasma. These RF powers were selected so as to equalize the ion saturation current density at approximately 1 $\mathrm{mA} / \mathrm{cm}^{2}$ in both argon and oxygen plasmas. A water-cooled sample stage was set $95 \mathrm{~mm}$ downstream from the antenna. The substrate stage was kept electrically floating and the potential gap between the floating electrode and the argon plasma was measured to be $12 \mathrm{~V}$ by the Langmuir probe method. The temperature of the sample was kept below $323 \mathrm{~K}$ during plasma exposure. The plasma exposure time was varied as an experimental parameter.

In addition, in a comparative experiment, the polymer surface was exposed to $172 \mathrm{~nm}$ vacuum UV (VUV) light using a Xe excimer lamp (lamp type: 172/120 Z (HV), Heraeus Noblelight $\mathrm{GmbH}$, Germany). VUV exposure was carried out in a 100 $\mathrm{Pa}$ oxygen atmosphere at $40 \mathrm{~mW} / \mathrm{cm}^{2}$ for $10 \mathrm{~min}$.

\section{2. Analysis of treated samples}

Immediately after the plasma treatment, the water contact angle of the sample surface was measured by the sessile drop method. The chemical bonding states of treated surfaces were also analyzed by XPS with a $4 \mathrm{kV}$ acceleration voltage (AXIS-HSi, KRATOS Shimadzu Co., Ltd., Japan). Monochromatic $\mathrm{Al} \mathrm{K} \alpha$ was used as an X-ray source. An atomic force microscope (SPM-9600, Shimadzu) and a UV-Vis-NIR spectrophotometer (V-670, JASCO Corp., Japan) were used to evaluate the surface morphology and optical transmission of the sample, respectively.

Moreover, to examine the suitability of the hydrophilized amorphous perfluoropolymer for applications involving cell cultivation, $1 \times 10^{4}$ human epithelioid carcinoma (HeLa) cells were seeded on treated polymer samples immersed in a 35-mm-well culture dish filled with Dulbecco's modified Eagle medium (high-glucose) (invitrogen Corp., U.S.A.) supplemented with $10 \%$ fetal bovine serum (invitrogen), $100 \mu \mathrm{g} / \mathrm{ml}$ streptomycin (invitrogen), 100 units $/ \mathrm{ml}$ penicillin (invitrogen), and $100 \mu \mathrm{g} / \mathrm{ml}$ kanamycin (invitrogen). The cells were cultured for 7 days using an integrated cell culture observation device (BioStation CT, Nikon Instruments Co., Ltd., Japan) in an environment maintained at $37^{\circ} \mathrm{C}$ and $85 \%$ humidity. Throughout the entire cultivation experiment, the cells were regularly observed under a phase contrast light 
microscope equipped inside the device.

\section{Results and Discussion}

3. 1. Water contact angle measurements

First, the hydrophilization of Cytop ${ }^{\mathrm{TM}}$ surfaces by argon and oxygen plasmas was examined. Although oxygen plasma exposure is often adopted for the hydrophilization of polymer surfaces, in the present study, the predominant surface reaction that occurred for the case of oxygen plasma treatment was etching rather than hydrophilization. In addition, exposure to $172 \mathrm{~nm}$ VUV irradiation did not modify the hydrophilicity of the polymer surface. In contrast, argon plasma exposure markedly decreased the water contact angle of the surface. On the basis of these results, the surface hydrophilization was thereafter investigated using argon plasma.

The effect of argon plasma exposure time on the water contact angle of the treated sample surface was examined, as shown in Fig. 1. In the first $60 \mathrm{~s}$ exposure to argon plasma, the water contact angle decreased rapidly from $110^{\circ}$ to $60^{\circ}$. After further exposure up to $180 \mathrm{~s}$, the water contact angle gradually decreased to $30^{\circ}$ and saturated upon further exposure. In addition, it is noteworthy that hydrophobic recovery was hardly observed in the present study.

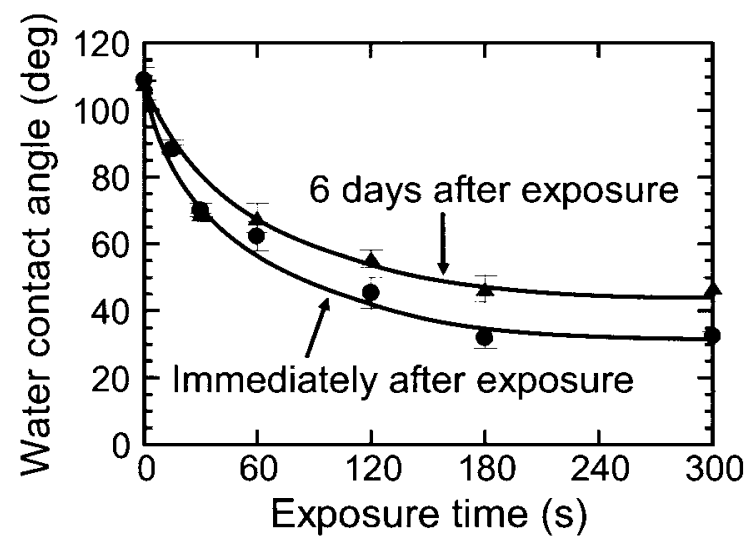

Fig. 1 Effect of Ar plasma exposure time on water contact angle of Cytop ${ }^{\mathrm{TM}}$. The plasma conditions were an RF power of $25 \mathrm{~W}(13.56 \mathrm{MHz})$ and a total Ar gas pressure of $4 \mathrm{~Pa}$. Error bars represent one standard deviation.

The observed hydrophobic recovery was no more than $15^{\circ}$ even after storage for 6 days; moreover, stable hydrophilicity was confirmed even after storage for one month. Such long-term stability can be explained by the poor mobility of the polymeric chains owing to their inherent cyclic structures, which prevents the surface reorientation.

3. 2. Effects of argon plasma treatment on chemical bonding state of surface

XPS C1s, O1s, and F1s spectra were measured to identify the composition and chemical bonding state of the polymer surface. In particular, the chemical shifts in $\mathrm{Cls}$ and Ols spectra were analyzed in detail. An overlay plot of C1s spectra obtained from the oxygen-plasma- and argonplasma-treated samples and the untreated control sample is shown in Fig. 2. The spectrum obtained from the oxygen-plasma-treated sample was almost the same as that from the untreated surface except for the additional peak observed at $285 \mathrm{eV}$, which corresponds to the $\mathrm{C}-\mathrm{C}$ bond. On the other hand, the spectrum obtained from the argonplasma-treated sample is markedly different from that of the untreated surface. Thus, the changes in the chemical bonding state of the argon-plasmatreated surface are consistent with the large change in water contact angle.

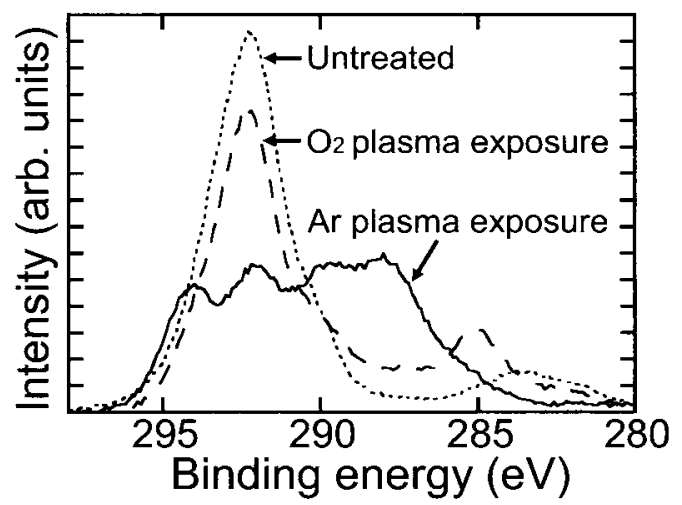

Fig. 2 XPS C1s spectra obtained from untreated, oxygen-plasma-treated and Ar-plasma-treated Cytop ${ }^{\text {TM }}$ surfaces. Dotted line: untreated control sample, dashed line: oxygen-plasma-treated sample, and solid line: Ar-plasma-treated sample. The conditions for the oxygen plasma were $R F$ power $=100 \mathrm{~W}(13.56 \mathrm{MHz})$, total $\mathrm{O}_{2}$ gas pressure $=4 \mathrm{~Pa}$ and exposure time $=180 \mathrm{~s}$. For Ar plasma RF power $=25 \mathrm{~W}(13.56 \mathrm{MHz})$, total $\mathrm{Ar}$ gas pressure $=4 \mathrm{~Pa}$, and exposure time $=40 \mathrm{~s}$.

To clarify the type of functional groups responsible for the modification of hydrophilicity by exposure to argon plasma, C1s and O1s XPS spectra were measured for four samples with different treatment times and storage times in the atmosphere, and the chemical shift peaks in the 

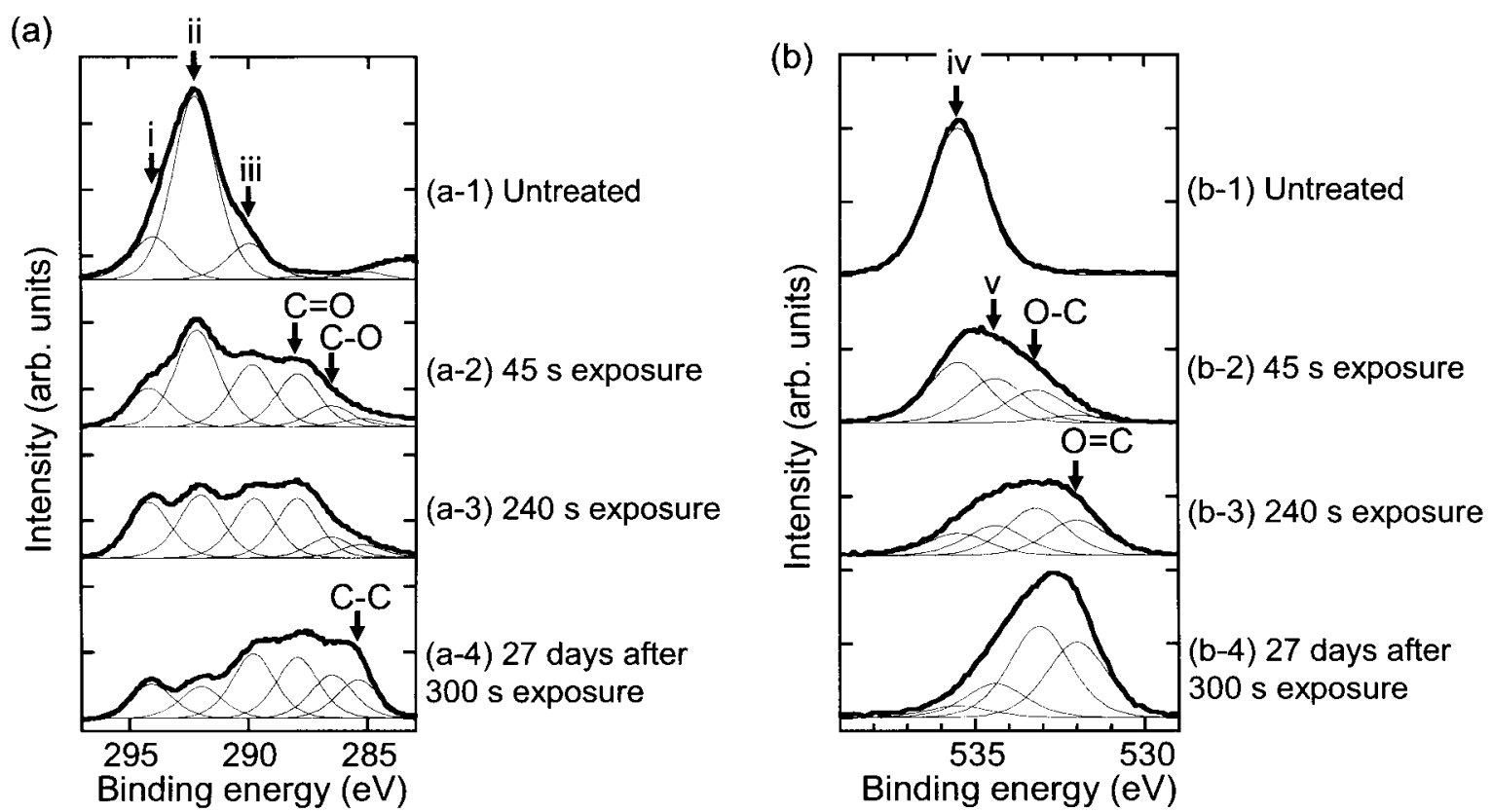

Fig. 3 XPS spectra obtained from untreated and treated Cytop ${ }^{\mathrm{TM}}$ surfaces. (a) C1s spectra and (b) O1s spectra. With increasing exposure time, the original structure of Cytop ${ }^{\mathrm{TM}}$, corresponding to peaks $\mathrm{i}$, ii, iii, and iv, was decomposed (peak $\mathrm{v}$ is considered to be $\mathrm{CF}-\mathrm{O}-\mathrm{CF}_{2}$, one of the structures of decomposed Cytop ${ }^{\mathrm{TM}}$ ) and $\mathrm{C}-\mathrm{O}, \mathrm{C}=\mathrm{O}$, and $\mathrm{C}-\mathrm{C}$ structures were produced. Each peak was fitted with a Gaussian-Lorentzian mixed function with a full width at half maximum of $2.1 \mathrm{eV}$. The plasma conditions were $\mathrm{RF}$ power $=25 \mathrm{~W}(13.56 \mathrm{MHz})$ and Ar gas pressure $=4 \mathrm{~Pa}$.

spectra were separated, as shown in Fig. 3. Three samples were measured within $4 \mathrm{~h}$ after the plasma treatment for 0,45 , and $240 \mathrm{~s}$, whereas another sample was measured after 27 days of storage following treatment for $300 \mathrm{~s}$. As shown in Fig. 3 (a-1), the C1s spectrum obtained from the untreated surface is composed of three dominant peaks at $294.1 \mathrm{eV}$ (i), $292.1 \mathrm{eV}$ (ii), and $289.9 \mathrm{eV}$ (iii). These peaks originate from the bonds that exist in native Cytop ${ }^{\mathrm{TM}}$.

Figures 3 (a-2) and (a-3) show that argon plasma exposure results in a decrease in the intensity of the original three peaks and the appearance of new peaks at 288.0 and $286.6 \mathrm{eV}$, which are attributed to $\mathrm{C}=\mathrm{O}$ and $\mathrm{C}-\mathrm{O}$ bonds, respectively [10]. Such spectral changes indicate that C-F bonds were broken during argon plasma exposure and then hydrophilic groups containing carbon-oxygen bonds were formed on the treated surface. The decrease in the surface concentration of fluorine atoms was also confirmed by the quantitative analysis of XPS data. The atomic composition of fluorine in the original polymer was measured to be $59 \%$, whereas it decreased to $48-49 \%$ after 45 and $240 \mathrm{~s}$ of plasma treatment. As shown in Fig. 3 (a-4), the modified chemical bonding state was stable even after long-term storage in air for 27 days, although some increase in $\mathrm{C}-\mathrm{C}$ peak intensity at $285.3 \mathrm{eV}$ owing to the adsorption of organic contaminants was observed [11]. Similarly, the peak separation of the O1s XPS spectra was carried out. As shown in Fig. 3 (b-1), the Ols spectrum obtained from the untreated polymer exhibited a single peak at $535.5 \mathrm{eV}$ (iv), which is attributed to $\mathrm{C}-\mathrm{O}-\mathrm{C}$ bonds in the original polymer structure. As can be seen in Fig. 3 (b-2), the intensity of this peak was decreased by $45 \mathrm{~s}$ plasma treatment, which caused the appearance of two new peaks. Peak at $534.4 \mathrm{eV}(\mathrm{v})$ is assigned to CF-O-CF ${ }_{2}$ [12], which was formed in the degraded amorphous perfluorocarbon polymer. The other peak at $533.2 \mathrm{eV}$ is ascribed to the $\mathrm{O}-\mathrm{C}$ bond [13] As shown in Fig. 3 (b-3), when the exposure time was $240 \mathrm{~s}$, peak iv further decreased in intensity and another peak corresponding to the $\mathrm{O}=\mathrm{C}$ or $\mathrm{C}-\mathrm{O}-\mathrm{C}$ structure was observed at $532.0 \mathrm{eV}$ [13]. In the XPS spectrum obtained from the sample after storage for 27 days after $300 \mathrm{~s}$ plasma treatment, peak iv nearly disappeared and two peaks corresponding to $\mathrm{O}-\mathrm{C}$ and $\mathrm{O}=\mathrm{C}$ bonds became dominant, as shown in Fig. 3 (b-4). Thus, the incorporation of hydrophilic groups by the argon plasma treatment was confirmed by XPS analysis. 
3. 3. Effects of argon plasma treatment on physical properties

To obtain further insight into the changes in physical properties of the plasma-treated polymer surface, the surface morphology was observed by AFM. As shown in Fig. 4, the average surface roughness was $0.31 \mathrm{~nm}$ for the untreated control sample and $0.32 \mathrm{~nm}$ for the sample treated with argon plasma for 60 s. Thus, no topographical modification was observed upon argon plasma exposure. Although surface roughness is known to have an effect on hydrophilicity [14], it does not play a role in the present study.
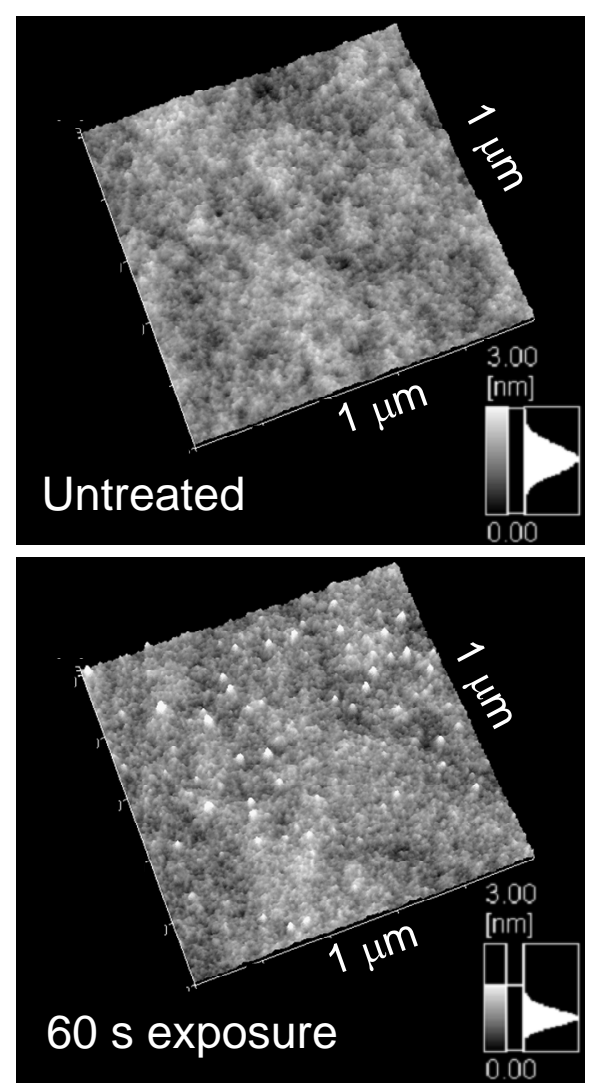

Fig. 4 AFM images of amorphous perfluoropolymer surfaces. The average roughnesses were $0.31 \mathrm{~nm}$ for the untreated sample (upper) and $0.32 \mathrm{~nm}$ for sample treated for $60 \mathrm{~s}$ (lower). The surface roughness of the treated sample was not markedly different from that of the untreated control sample. The plasma conditions were an RF power of $25 \mathrm{~W}(13.56 \mathrm{MHz})$ and an Ar gas pressure of $4 \mathrm{~Pa}$.

Subsequently, optical transmission spectra of the perfluoropolymer in the UV-Vis region were measured for the sample treated for $300 \mathrm{~s}$ and the untreated control sample. In this experiment, a $2-\mu \mathrm{m}$-thick polymer layer coated on 1-mm-thick high-purity synthetic silica glass with high transparency in the UV region was used as a sample. As shown in Fig. 5, no fine characteristic structures were observed other than the fringe pattern in both spectra, and the high transmittance in the visible-light region was hardly changed. However, the degradation of transmittance in the UV region was observed after the exposure to plasma. The result indicates that a thin layer with a modified refractive index was formed on the polymer surface through the cross-linking of free radicals or chain scission induced by the exposure to plasma.

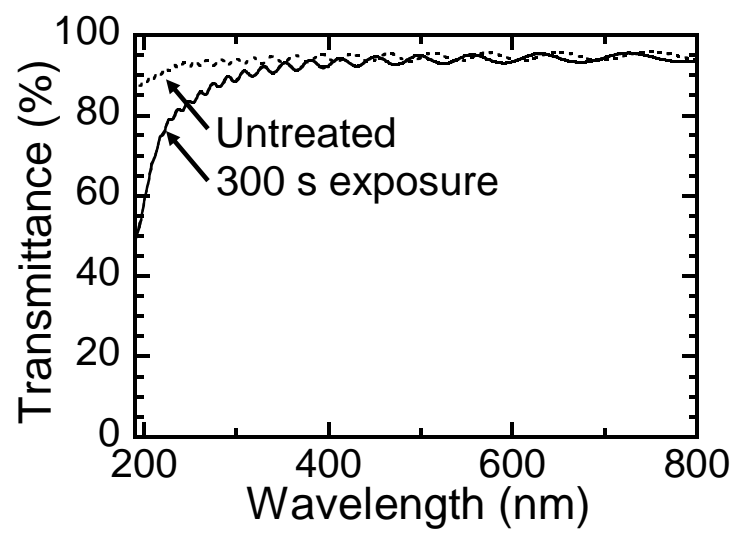

Fig. 5 Transmission spectrum of amorphous perfluoropolymer films with/without $\mathrm{Ar}$ plasma exposure. Dotted line: untreated sample, solid line: sample exposed to Ar plasma. After 300 s exposure, the transmittance decreased below wavelength of $400 \mathrm{~nm}$. The conditions for the Ar plasma were RF power $=25$ $\mathrm{W}$ and pressure $=4 \mathrm{~Pa}$.

Here, the effects of argon plasma exposure on the modification of the amorphous perfluoropolymer surface are briefly discussed. Generally, argon is inert, and therefore, the main effect of argon plasma treatment results from the transfer of physical energy through the interaction of energetic particles and UV photons with the polymer surface. Unfortunately, the contributions of ion bombardment and VUV photon irradiation could not be discussed individually from the present experimental results. Although the samples were set on an electrically floating stage in the plasma, ions impinging on the samples can be accelerated to approximately $10 \mathrm{eV}$ in accordance with the results of Langmuir probe measurement, resulting in the structural modification of the polymer surface. On the other hand, $172 \mathrm{~nm}$ VUV irradiation did not modify the polymer surface, as 


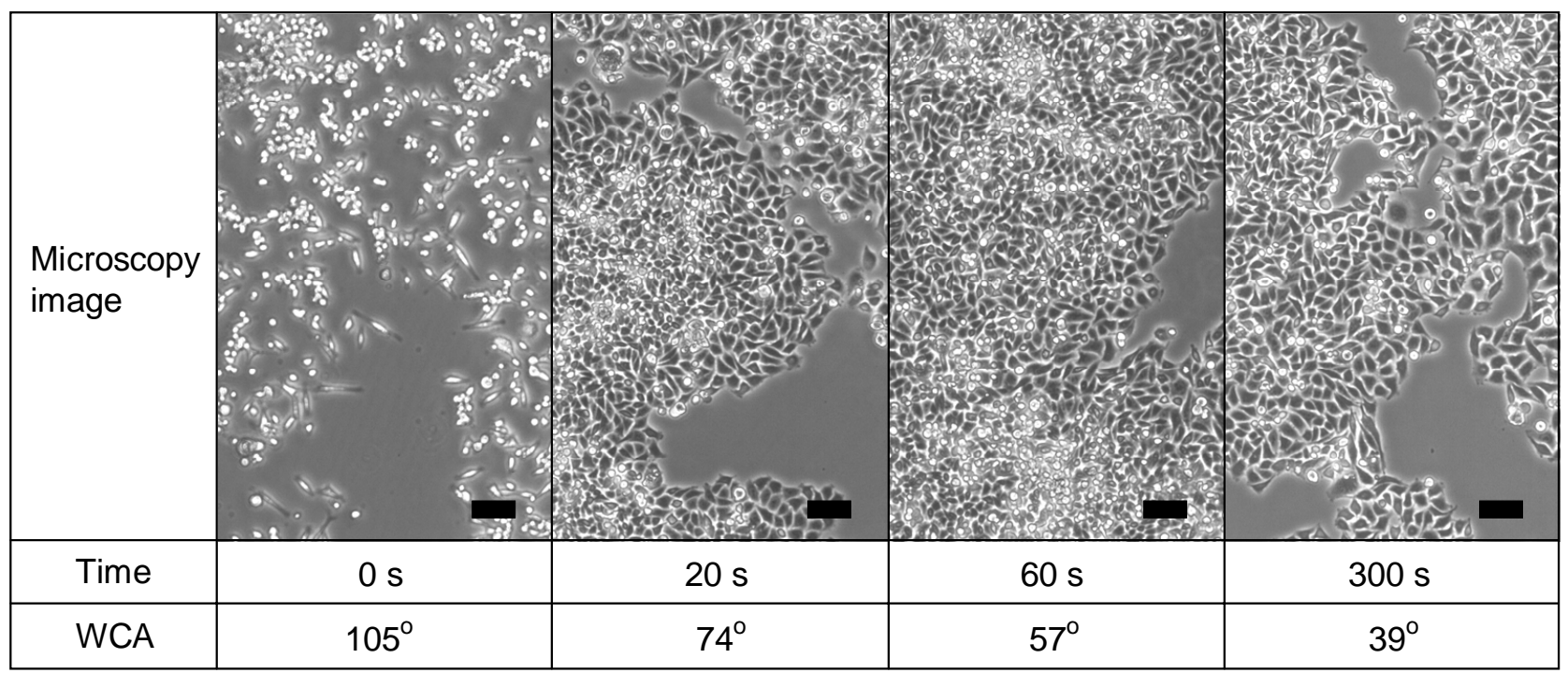

Fig. 6 Effect of exposure time and water contact angle (WCA) on density of cultured HeLa cells. The plasma conditions were an RF power of $25 \mathrm{~W}(13.56 \mathrm{MHz})$ and an Ar gas pressure of $4 \mathrm{~Pa}$. Scale bars represent $100 \mu \mathrm{m}$.

mentioned in section 3.1., but argon plasma contains photons with a shorter wavelength than $172 \mathrm{~nm}$ that can directly break the C-C, C-F, and $\mathrm{C}-\mathrm{O}$ bonds in Cytop ${ }^{\mathrm{TM}}[15]$. The exposure to argon plasma led to bond breaking, the formation of free radicals, and finally the incorporation of polar groups such as $\mathrm{C}=\mathrm{O}$ and $\mathrm{C}-\mathrm{OH}$ as a result of the reaction with oxygen and water from the atmosphere after removed from the plasma reactor. Such chemical modification is expected to be the main origin of the modified surface hydrophilicity; the surface morphology has little effect on the surface hydrophilicity, as observed in the present study.

\section{4. Cell adhesion}

As shown in the phase-contrast images of Fig. 6 , improved adhesion and the proliferation of HeLa cells were observed for cells cultured on plasma-treated amorphous perfluoropolymer surfaces. The highest number of adhering cells and the highest growth rate were observed on the sample exposed to argon plasma for $60 \mathrm{~s}$, followed by those with exposure times of 20 and $300 \mathrm{~s}$. In contrast, on the untreated surface, most of the seeded cells exhibited poor adhesion, maintained a circular shape and were easily washed away by a single mild rinse with phosphate-buffered saline. This result corresponds to the well-known fact that a water contact angle of approximately $60^{\circ}$ is suitable for the adhesion of cells to a solid surface
[16-18]. Thus, owing to the hydrophilization process, cell cultivation was enabled on the polymer. This achievement is important, particularly when the amorphous perfluoropolymer is to be applied in cell engineering research, which often requires the precise imaging of cells.

\section{Conclusion}

The surface hydrophilization of an amorphous perfluoropolymer using low-pressure argon plasma was studied. It was found that exposure to argon plasma can decrease the water contact angle from $110^{\circ}$ to $30^{\circ}$, and little hydrophobic recovery was observed even after one month of storage. XPS, AFM, and UV-vis-NIR spectroscopy were employed to evaluate the changes in the surface properties after argon plasma exposure. The main origin of the hydrophilization was found to result from the incorporation of hydrophilic groups and not from the change in surface topography. Optical transmittance was degraded in the UV region upon argon plasma treatment, possibly owing to the formation of a thin modified layer on the surface, but was hardly changed in the visible light region. Moreover, the regular proliferation of cells were confirmed when HeLa cells were cultured on plasma-treated amorphous perfluoropolymer surfaces. The simple and robust surface process proposed in this study, which enables the surface hydrophilization of the amorphous perfluoropolymer with long-term stability, is expected to be 
useful for the introduction of this polymer into various $\mu$ TAS and biodevice applications.

\section{References}

1. J. C. McDonald and G. M. Whitesides, Acc. Chem. Res., 35 (7) (2002) 491.

2. H. Lu, M. A. Schmidt, and K. F. Jensen, Lab on a Chip, 1 (2001) 22.

3. Asahi Glass Co., Ltd., CYTOPтм Tech. Data 1, 2007.

4. A. Egner, M. Schrader, and S. W. Hell, Opt. Commun., 153 (1998) 211.

5. K. Yamamoto and G. Ogawa, J. Fluorine Chem., 126 (2005) 1403.

6. K. Obata, K. Sugioka, N. Shimazawa, and K. Midorikawa, Appl. Phys., A 84 (2006) 251.

7. R. Wilke and S. Büttgenbach, Biosens. Bioelectron., 19 (2003) 149.

8. K. W. Oh, A. Han, S. Bhansali, and C. H. Ahn, J. Micromech. Microeng., 12 (2002) 187.

9. T. Ono, T. Akagi, and T. Ichiki J. Appl. Phys., 105 (2009) 013314.

10. J. Laine, P. Stenius, G. Carlsson, and G. Ström,
Cellulose, 1 (1994) 145.

11. T. Ichiki, H Oshio, and Y Horiike, J. Surf. Anal., 5 (1999) 2.

12. B. Parekh, S. Zheng, A. Entenberg, T. Debies, and G. A. Takacs, J. Adhesion Sci. Technol., 21 (10) (2007) 983.

13. M. T. Martínez, M. A. Callejas, A. M. Benito, M. Cochet, T. Seeger, A. Ansón, J. Schreiber, C. Gordon, C. Marhic, O. Chauvet, J. L. G. Fierro, and W. K. Maser, Carbon, 41 (2003) 2247.

14. R. N. Wenzel, Ind. Eng. Chem., 28 (1936) 988. 15. R. H. French, R. C. Wheland, W. Qiu, M. F. Lemon, E. Zhang, J. Gordon, V. A. Petrov, V. F. Cherstkov, and N. I. Delaygina, J. Fluorine Chem., 122 (2003) 63.

16. P. B. van Wachem, A. H. Hogt, T. Beugeling, J. Feijen, A. Bantjes, J. P. Detmers, and W. G. van Aken, Biomaterials, 8 (1987) 323.

17. J. H. Lee, G. Khang, J. W. Lee, and H. B. Lee, J. Colloid Interface Sci., 205 (1998) 323.

18. Y. Tamada and Y. Ikada, Polymer, 34 (1993) 2208. 